\title{
Lowest PAPR Selection Technique from SLM and PTS with One IFFT Block in Wireless MC-CDMA Communication Systems
}

\author{
Mesri Mokhtaria, Merah Hocine, and Tahkoubit Khaled
}

\begin{abstract}
MC-CDMA is the most promising technique for high bit rate and capacity transmission in wireless communication. One of the challenging issues of MC-CDMA system is the very high PAPR due to the large number of sub-carriers which reduces the system efficiency. In our work, a new (SLM-PTS) method is suggested with one IFFT block for PAPR reduction in MC-CDMA downlink transmission. The new method has showed a significant improvement in PAPR reduction performance and system complexity. SLM-PTS is compared to PTS and SLM at the term of PAPR reduction, as well as it is compared to the original system at the term of bit-error-rate (BER), firstly, without and then using the linear amplifier (SSPA).
\end{abstract}

Index Terms-IFFT, MC-CDMA, PAPR, PTS, SLM, SSPA.

\section{INTRODUCTION}

It is well known that high bit rate transmission is required for high quality broadband wireless communication. The $21^{\text {st }}$ century systems ( $3^{\text {rd }}$ generation and beyond) have to support a large range of multimedia services such as speech, images and data with different and variable bit rates up to $2 \mathrm{Mbits} / \mathrm{s}$.

Future radio communication systems will have to accommodate high data rate while allowing a great mobility to the users. In order to achieve this goal, new signal processing techniques must be investigated. . In this paper, one of the techniques under current significant research, namely the MC-CDMA (Multi-Carrier Code Division Multiple Access) technique, is suggested.

The code-division multiple access system has higher frequency efficiency than conventional time division multiple access (TDMA) or frequency division multiple access (FDMA) systems. However, the capacity is limited by the inter-chip interference (ICI) and multiple-access interference (MAI). Besides, multi-carrier (MC) transmission technique has many advantages such as high bandwidth efficiency, excellent frequency diversity, and high speed parallel transmission. When subcarrier number ischosen appropriately, there is only flat fading that has no ICI in each sub-channel. Recently, considerable interests are focused on the combined scheme of OFDM and CDMA [1].

MC-CDMA is a very attractive technique for a high speed data transmission over the multipath fading channels. High Peak to Average Power Ratio (PAPR) of the transmitted

Manuscript received August 4, 2016; revised November 4, 2016.

Mesri Mokhtaria and Tahkoubit Khaled are with Electronics Department, Faculty of Technology, University Amar Telidji, BP 37G, Laghouat 03000, Algeria (e-mail: meradmesri@yahoo.fr.).

Merah Hocine is with Electronics Department, Faculty of Technology, University Ferhat Abbas, Setif, 019000, Algeria. signal is a serious problem in multicarrier systems (MC), such as Orthogonal Frequency Division Multiplexing (OFDM), or in Multi-Carrier Code Division Multiple Access (MC-CDMA) systems, due to large number of subcarriers This effect is possible to reduce with some PAPR reduction techniques. High Power Amplifier (HPA) have big influence on the behavior of the system, which results in a large degradation of performance, i.e. increase of both the bit error rate (BER) and the out-of-band radiation (spectral spreading). Since decades, many solutions have been proposed to solve this problem. Some of them compensate for non-linearities at the transmitter side and some of them carry out the processing at the receiver side. It is necessary first to distinguish between processing on the amplification function and processing on the signal itself.

To reduce the PAPR, various techniques have been proposed in literature including the clipping and filtering technique [2], [3], the Partial Transmit Sequences (PTS) [4], [5], the Selective Mapping (SLM) method [6], and Tone Reservation (TR) [7], etc...

Our work is organized as follows. In Section two, general concepts of a MC-CDMA system are explained; definition and mathematical expression of the PAPR are also given. In Section three a new (SLM-PTS) method for PAPR reducing purposes is proposed. The simulation results are described in section four. The last Section provides some conclusions.

\section{MC-CDMA SYSTEMS}

In an MC-CDMA system [8, 9] a block of M information symbols from each active user are spread in the frequency domain into $\mathrm{N}=\mathrm{L} . \mathrm{M}$ subcarriers, where $\mathrm{L}$ represents the spreading factor. This is accomplished by multiplying every symbol of the block for user $\mathrm{k}$, where $\mathrm{k} \in\{0,1, \ldots, \mathrm{L}-1\}$, by a spreading code $\left\{\mathrm{C}_{\mathrm{l}}{ }^{(\mathrm{k})}, \mathrm{l}=0,1, \ldots, \mathrm{L}-1\right\}$, selected from a set of $\mathrm{L}$ orthogonal sequences, thus, allowing a maximum of $\mathrm{L}$ simultaneous users to share the same radio channels. The spreading codes are the usual Walsh-Hadamard (WH) sequences, which are the columns of the Hadamard matrix of order $L, C_{L}$. If $L$ is a power of 2 , the Hadamard matrix is constructed recursively as

$$
\begin{aligned}
C_{2} & =\left(\begin{array}{cc}
1 & 1 \\
1 & -1
\end{array}\right) . \\
C_{n} & =C_{n / 2} * C_{2} .
\end{aligned}
$$

where the symbol «*» denotes de Kronecker tensor product.

In the downlink transmitter, each spread symbol of every active user is added to the spread symbols of the remaining 
active users, and the resulting sums are interleaved to form a set of $N=L . M$ complex amplitudes as follows:

$$
X(M . l+m)=\sum_{k=1}^{N_{u s r}} C_{l}^{(k)} \times a_{m}^{(k)} .
$$

where $N_{u s r}$ is the total number of active users and $\left\{\mathrm{a}_{\mathrm{l}}{ }^{(\mathrm{k})}, \mathrm{m}=\right.$ $0,1, \ldots, M-1$, are the data symbols in the block for the $\mathrm{k}^{\text {th }}$ active user.

After Inverse Fast Fourier Transform (IFFT) operation, the time domain signal $x=[x(0), x(1), \ldots, x(N . Q-1)]$ is

$$
x(n)=\frac{1}{N \cdot Q} \sum_{k=0}^{N-1} X(k) \cdot e^{j \frac{2 \pi n k}{N \cdot Q}} .
$$

where $n=0,1, \ldots, \mathrm{N} . \mathrm{Q}-1$; the oversampling factor $\mathrm{Q}$ is an integer.

\section{A. Peak to Average Power Ratio (PAPR)}

The PAPR of a complex signal $\mathrm{x}(\mathrm{n})$ can be defined as the ratio of the peak envelope power to the average envelope power:

$$
P A P R=\frac{\underset{0 \leq n \leq N \cdot Q-1}{\operatorname{Max}}|x(n)|^{2}}{E\left[|x(n)|^{2}\right]}
$$

where $E[$.$] represents the expectation operation.$

The Complementary Cumulative Distribution Functions CCDF formula that approximates the PAPR of a multicarrier signal with Nyquist sampling rate is derived from the central limit theorem [10] and is given by:

$$
\begin{aligned}
\operatorname{CCDF}\left(P A P R_{0}\right) & =\operatorname{Prob}\left(\operatorname{PAPR}(x)>P A P R_{0}\right) \\
& =1-\left(1-e^{-P A P R_{0}}\right)^{N \cdot Q} .
\end{aligned}
$$

where $P A P R_{0}$ indicates the threshold value.

\section{B. Solid State Power Amplifier (SSPA)}

The SSPA [11] demonstrates non-linear characteristics, as it causes distortion of the signals, particularly the high PAPR values. Therefore, the BER performance of the system is decreased. The input and output signals of the SSPA are defined as:

$$
\begin{gathered}
x^{\prime}(n)=|x(n)| e^{j \theta(n)} . \\
r(n)=A_{S S A P}(|x(n)|) e^{j\left\{\theta(n)+\vartheta_{S S A P}(|x(n)|)\right\}} .
\end{gathered}
$$

Respectively, $|\mathrm{x}(\mathrm{n})|$ is the input signal amplitude, $\theta(\mathrm{n})$ is the input signal phase, $A_{\text {SSAP }}(|x(n)|)$ is the output signal amplitude and $\vartheta_{\text {SSAP }}(|x(n)|$ is the output phaseresponse. Amplitude/amplitude (AM/AM) and amplitude/phase $(\mathrm{AM} / \mathrm{PM})$ characteristics of the SSPA are defined as:

$$
\begin{gathered}
A_{S S A P}(|x(n)|)=\frac{|x(n)|}{\left[1+\left(|x(n)| / A_{0}\right)^{2 p}\right]^{1 / 2 p}} . \\
\vartheta_{S S A P}(|x(n)|)=0 .
\end{gathered}
$$

where $A_{0}$ is the output saturation amplitude and $\mathrm{p}$ is the smoothness control coefficient. The operating point of the SSPA is determined by the Input Back-Off (IBO) parameter and is expressed as:

$$
I B O=10 \log _{10}\left(\frac{P_{\max }}{P_{\text {ave }, \text { in }}}\right) .
$$

where $P_{\text {ave,in }}$ is the mean power of the input signal and $\mathrm{P}_{\max }$ is the peak power of the SSPA.

Fig. 1 represents the MC-CDMA downlink transmitter.

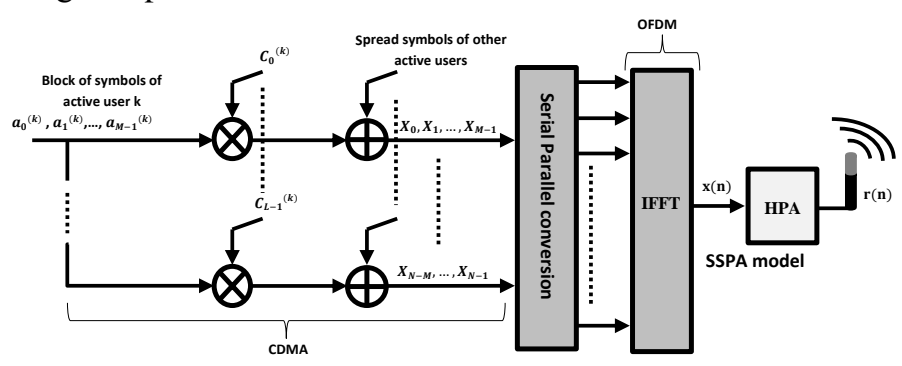

Fig. 1. MC-CDMA downlink transmitter.

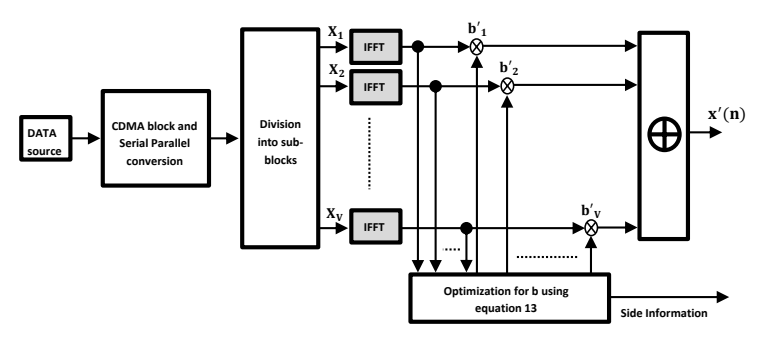

Fig. 2. Block diagram of the PTS techniques.

\section{The Partial Transmit Sequences (PTS) technique}

In the PTS technique [12], the input data block $\mathrm{X}$ is partitioned into $\mathrm{V}$ disjoint subblocks $\quad \mathrm{X}_{\mathrm{m}}=\left[\mathrm{X}_{\mathrm{m}, 0}, \mathrm{X}_{\mathrm{m}, 1}, \ldots, \mathrm{X}_{\mathrm{m}, \mathrm{N}-1}\right] \quad, \quad \mathrm{m}=$ $1,2, \ldots, \mathrm{V}$ such that:

$$
X=\sum_{m=1}^{V} X_{m}
$$

Complex phase factors are, $b_{m}=e^{j 2 \pi \frac{q m}{w}}, q_{m}=$ $0,1, \ldots W-1$, where $W$ is the number of allowed phase factors. The set of the phase factors shall be written as a vector $b=\left[b_{1}, b_{2}, \ldots, b_{V}\right]^{T}$. The time-domain signal after combining is given by:

$$
X^{\prime}(b)=\sum_{m=1}^{V} b_{m} X_{m}
$$

After Inverse Fast Fourier Transform (IFFT) operation, the time domain signal $x^{\prime}(b)=\left[x^{\prime}{ }_{0}(b), x^{\prime}{ }_{1}(b), \ldots, x^{\prime}{ }_{N . Q-1}(b)\right]$ is:

$$
x^{\prime}(b)=\sum_{m=1}^{V} b_{m} x_{m}
$$

The objective is to find the phase factors with the aim of minimizing PAPR. This is related to the minimization of:

$$
b^{\prime}=\operatorname{argmin}\left(\frac{\operatorname{Max}_{0 \leq n \leq N \cdot Q-1}\left|x_{n}^{\prime}(b)\right|^{2}}{E\left[\left|x_{n}^{\prime}(b)\right|^{2}\right]}\right)
$$

Fig. 2 shows the block diagram of PTS technique.

In our work only one subblock of IFFT is retained to simplify this technique; the subblocks of data should be 
chosen as follows:

$$
\left\{\begin{array}{c}
X_{1}=\left[X_{0}, \ldots, X_{\left(\frac{N}{V}\right)}, 0, \ldots, X_{\left(\frac{2 . N}{V}\right)}, 0, \ldots, X_{\left(\frac{(V-1) . N}{V}\right)}, \ldots, 0\right] \\
X_{2}=\left[0, X_{1}, \ldots, X_{\left(\frac{N}{V}+1\right)}, \ldots, 0, X_{\left(\frac{2 . N}{V}+1\right)}, 0 \ldots, 0,0 \ldots, 0\right] \\
\cdot \\
\cdot \\
\left.X_{V}=\left[0, \ldots, X_{V-1}, 0, \ldots, X_{\left(\frac{N}{V}+V-1\right.}\right), 0, \ldots, X_{\left(\frac{2 . N}{V}+V-1\right)}, 0, \ldots, X_{(N-1)}\right]
\end{array}\right.
$$

The time domain signal is then defined through an Inverse Fast Fourier Transform (IFFT) operation, as:

$\left[\mathrm{x}_{1}(\mathrm{n}), \mathrm{x}_{2}(\mathrm{n}), \ldots, \mathrm{x}_{\mathrm{M}}(\mathrm{n})\right]^{\mathrm{T}}=$

G. $\left[x(n), x\left(n-\frac{N \cdot Q}{V}\right), \ldots, x\left(n-(V-1) \frac{N \cdot Q}{V}\right)\right]^{T}$

where

$\mathrm{G}=\left[\mathrm{e}^{\mathrm{j} \cdot 2 \cdot \pi \mathrm{k} \cdot \mathrm{m} / \mathrm{V}}\right]_{0 \leq \mathrm{k} \leq \mathrm{V}-1,0 \leq \mathrm{m} \leq \mathrm{V}-1}$

The new schema of diagram PTS will be as in Fig.3.

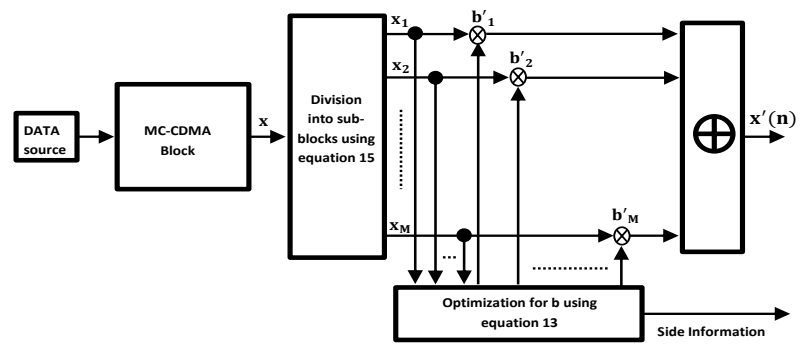

Fig. 3. New block diagram of the PTS techniques without IFFT subblocks.

\section{The Selected Mapping (SLM) Technique}

The block diagram of MC-CDMA system with SLM technique is shown in Figure 4. [13]; the input data sequences of each user with length $\mathrm{M}$ are first converted into $\mathrm{M}$ parallel data sequences and then each serial parallel converted output is multiplied with the spreading code with length L (CDMA Block). The out block of CDMA $\mathrm{X}=$ $\left[\mathrm{X}_{0}, \mathrm{X}_{1}, \mathrm{X}_{2}, \ldots, \mathrm{X}_{\mathrm{N}-1}\right]$ are multiplied by $\mathrm{U}-1$ different phase sequences $b^{\mathrm{u}}=\left[\mathrm{b}_{0}^{\mathrm{u}}, \mathrm{b}_{1}^{\mathrm{u}}, \mathrm{b}_{2}^{\mathrm{u}}, \ldots, \mathrm{b}_{\mathrm{N}-1}^{\mathrm{u}}\right]$ whose length is equal to the number of carriers before IFFT process resulting in U-1 modified data blocks $\mathrm{X} \cdot \mathrm{b}^{\mathrm{u}}=\left[\mathrm{X}_{0} \cdot \mathrm{b}_{0}^{\mathrm{u}}, \ldots, \mathrm{X}_{\mathrm{N}-1} \cdot \mathrm{b}_{\mathrm{N}-1}^{\mathrm{u}}\right]$.After the IFFT process, the PAPR is calculated (using equation 13) for $U-1$ phase rotated symbols sequences and one original sequence and then the symbol sequence with lowest PAPR is selected for transmission and the corresponding selected phase sequence.

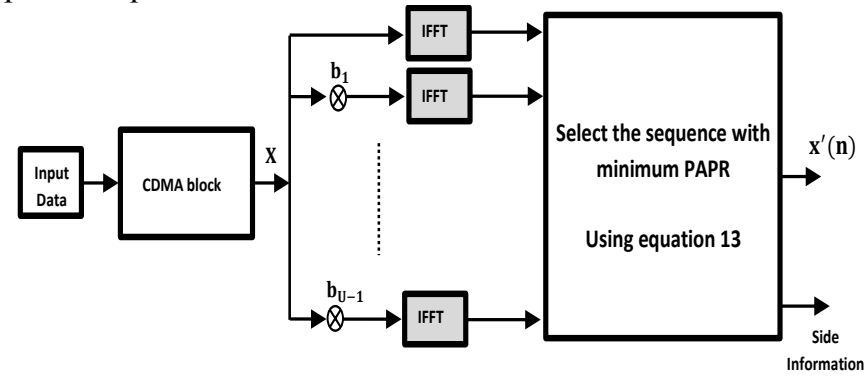

Fig. 4. Selected mapping (SLM) block DIAGRAM.

The problem of this technique is the number of the IFFT subblocks especially when the level of the parameter $U$ is increased. To avoid this problem, a new idea is investigated in this paper which consists in reducing the number of subblocks to the fullest extent possible (one block). Consequently, phase sequences are chosen for the value of the equation as follows:

$$
b^{u}(n)=\left\{\begin{aligned}
1, & n=2 \times k \\
e^{j \cdot 2 \cdot \pi \cdot n \cdot u / U}, & n=2 \times k+1
\end{aligned}\right.
$$

Where $\mathrm{k}$ is an integer value.

Therefore, the out block of CDMA $X$ is multiplied by $\mathrm{b}^{\mathrm{u}}(\mathrm{n})$.

The time domain signal is $\mathrm{x}^{\prime}\left(\mathrm{b}^{\mathrm{u}}\right)=\left[\mathrm{x}^{\prime}{ }_{0}\left(\mathrm{~b}^{\mathrm{u}}\right)\right.$, $\mathrm{x}^{\prime} 1 \mathrm{bu}, \ldots, \mathrm{x}^{\prime} \mathrm{N} . \mathrm{Q}-1 \mathrm{bu}$. It is obtained by Inverse Fast Fourier Transform (IFFT) as follows:

$$
\begin{aligned}
& x_{u^{\prime}}^{\prime}(n) \\
& =\frac{x(n)+x\left(n-N \cdot \frac{Q}{2}\right)}{2} \\
& +\frac{x\left(n-u^{\prime} \cdot N \cdot \frac{Q}{U}\right)-x\left(n-u^{\prime} \cdot N \cdot Q \cdot\left(\frac{1}{U}-\frac{1}{2}\right)\right)}{2}
\end{aligned}
$$

where

$$
u^{\prime}=\underset{1 \leq u \leq U-1}{\operatorname{argmin}}\left(\frac{\underset{0 \leq n \leq N \cdot Q-1}{\operatorname{Max}}\left|x_{u}^{\prime}(n)\right|^{2}}{E\left[\left|x_{u}^{\prime}(n)\right|^{2}\right]}\right)
$$

Fig. 5 represents the new SLM block.

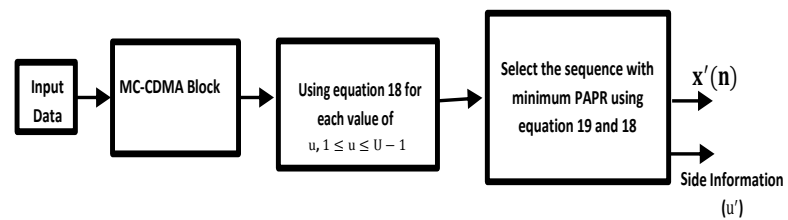

Fig. 5. New SLM block diagram without IFFT subblocks.

\section{COMBINED PTS AND SLM SCHEMES}

This is a complex model for PAPR reduction which contains two blocks for reducing PAPR which are SLM and PTS. An additional block allows us to select the corresponding method. $\mathrm{x}^{\prime}(\mathrm{n})$ outputs are responsible of choosing the type of method as well as the side information (SI) in order to carry the chosen signal and optimize parameters for each method at the receptor. Figure 6represents the new PTS-SLM model.

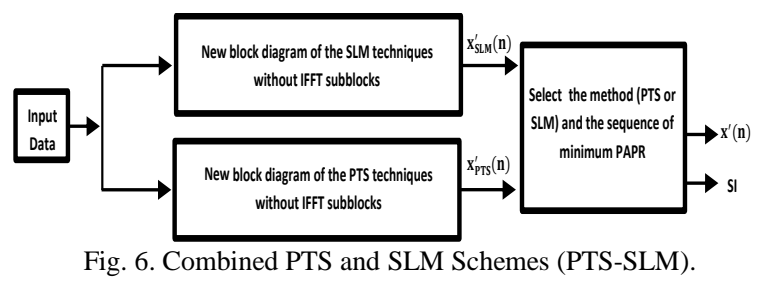

At the transmitter stage, the type of PAPR reduction method is determined by the following hypothesis:

$$
\begin{aligned}
& x^{\prime}(n) \\
& =\left\{\begin{array}{cc}
x_{S L M}^{\prime}(n) & P A P R\left(x_{S L M}^{\prime}\right)<P A P R\left(x_{P T S}^{\prime}\right) \\
x_{P T S}^{\prime}(n) & P A P R\left(x_{S L M}^{\prime}\right)>\operatorname{PAPR}\left(x_{P T S}^{\prime}\right)
\end{array}\right.
\end{aligned}
$$




\section{Simulation Results}

It has been used $\mathrm{N}_{\text {usr }}=16$ users, $\mathrm{N}=256$ sub-carriers, a Walsh Hadamard spreading code of length $\mathrm{L}=16$ and a 16-QAM modulation, for the MC-CDMA system. The oversampling factor of the system is $Q=4$. The number of sub-blocks $U=128,64,32,16,8,4$ are chosen for the SLM optimization whereas the different values of the parameters $W$ and $V .(V, W)=(4,4),(8,2),(4,2),(2,4)$ are chosen in the PTS optimization. In the new SLM-PTS optimization, $(U, V, W)=(64,4,2),(16,4,2),(64,4,4),(16,4,4)$ has been chosen. The SSPA is used with $I B O=12,6$ and $3 \mathrm{~dB}$ and smoothness factor $p=2$. The communication channel is Rayleigh fading. First the SLM, PTS and SLM-PTS methods are compared in terms of PAPR reduction performances in the MC-CDMA system. Moreover, the BER performance of the MC-CDMA system using SLM-PTS is shown when the SSPA and the linear amplifier are used.

\section{A. PAPR Reduction}

Fig. 7 illustrates the complementary cumulative distribution functions (CCDF) of the PAPR for original signals and other signals obtained by the SLM scheme. One can notice from the simulation results, that the SLM scheme for $U=128,64$ and 32 offers better PAPR reduction comparing to the SLM scheme with $U=16,8$ and 4. Given $U=64$ provides a PAPR reduction of $2.7 \mathrm{~dB}$ at $C C D F=$ $10^{-3}$, while, taking $U=4$ provides PAPR reduction of only $1 \mathrm{~dB}$ at $C C D F=10^{-3}$. It is known that the SLM scheme requires U.Q.N. $\log _{2}(\mathrm{Q} . \mathrm{N})+2$. U. $\mathrm{N}$ numbers of complex multiplications $\left(\mathrm{n}_{\mathrm{mul}}\right)$ and 2.U.Q.N. $\log _{2}(\mathrm{Q} . \mathrm{N})$ numbers of complex additions $\left(n_{\text {add }}\right)$, thus, for $U=4,8,16$, the SLM scheme the calculating complexity is $\mathrm{n}_{\mathrm{mul}}=43008,86016,172032$ $\mathrm{n}_{\mathrm{add}}=81920,163840,327680$ respectively.

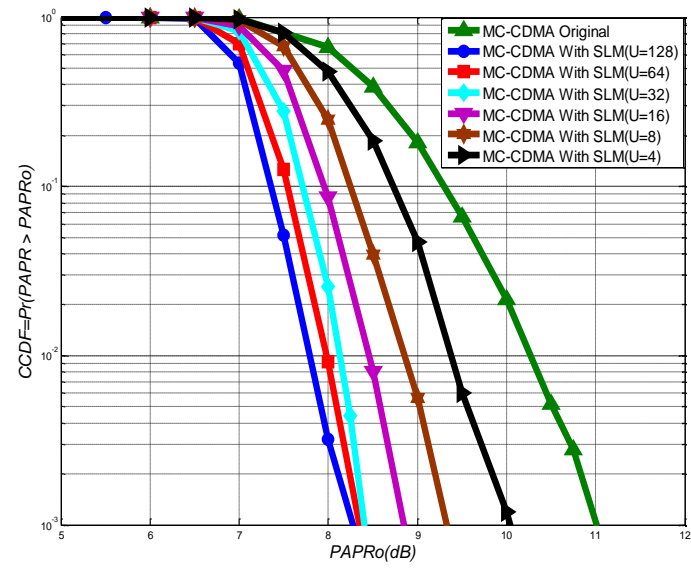

Fig. 7. PAPR reduction performance for SLM regarding different values of the parameter $\mathrm{U}$.

Fig. 8 shows the Complementary Cumulative Distribution Functions (CCDF) of the PAPR for original signals and other signals obtained by the PTS scheme. Increased values of $(V, W)$ offers better PAPR reduction. For example, given $(V, W)=(2,4)$ in PTS scheme provides only $0.75 d B$ of the PAPR at $C C D F=10^{-3}$, however, this value goes up to $3 d B$ with $(V, W)=(8,2)$. Therefore, PTS explores all the phase vectors to find the lowest value of the PAPR requiring $4^{4-1}=64$ and $2^{8-1}=128$ check in respectively.

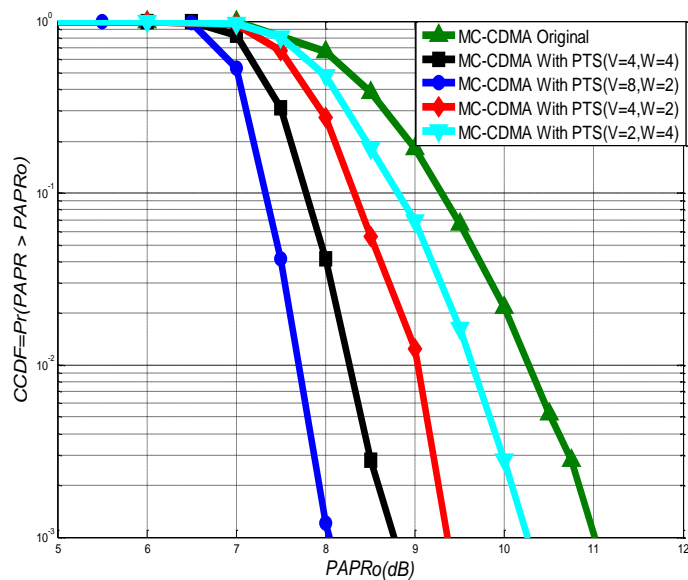

Fig. 8. PAPR reduction performances for PTS regarding different values of $\mathrm{V}$ and $\mathrm{U}$ parameters.

Fig. 9 shows the Complementary Cumulative Distribution Functions (CCDF) of the PAPR for original signals and other signals obtained by the SLM-PTS scheme. The SLM-PTS scheme for $(U, V, W)=(64,4,4)$ provides better PAPR reduction of $2.8 \mathrm{~dB}$ at $C C D F=10^{-3}$, comparing to $2.5 \mathrm{~dB}$ obtained with $(U, V, W)=(64,4,2)$.

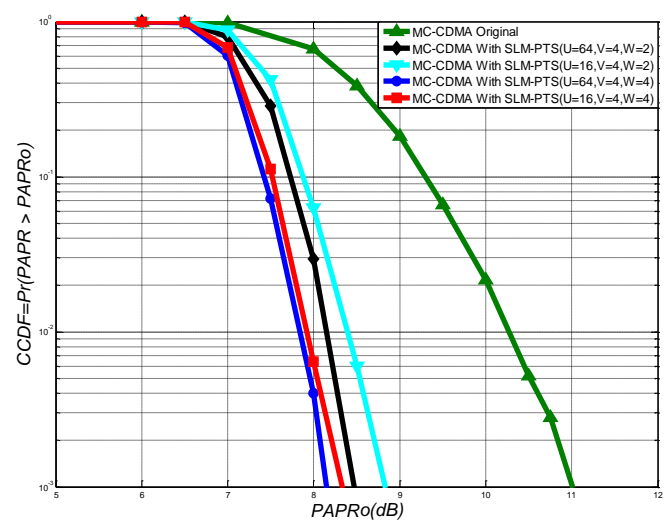

Fig. 9. PAPR reduction performance for SLM-PTS regarding different values of $\mathrm{U}, \mathrm{V}$ and $\mathrm{W}$ parameters.

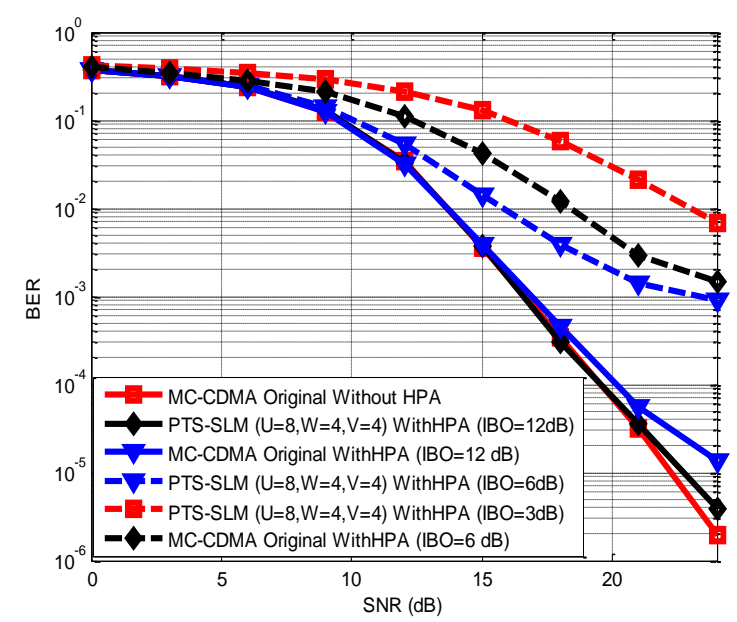

Fig. 10. BER performance with 16-QAM and different IBO over Rayleigh fading channel.

\section{B. BER Performance}

Figure. 10 shows the BER performance of the SLM-PTS scheme for 16-QAM and $(U, V, W)=(8,4,4)$ at input back off $I B O=12,6,3 d B$ in Rayleigh fading channels. The represented curves of Fig. 10 are obtained for the cases of the 
original signal transmitted without SSPA (MC-CDMA original without HPA), the SLM-PTS scheme with SSPA and the original signal transmitted with SSPA (MC-CDMA original with HPA) respectively. According to obtained results the IBO remains very important for the BER performance of the system. The new method (SLM-PTS) which is presented in this work, offers better performance of the MC-CDMA original with HPA. For example, $B E R_{\mathrm{SLM}-\mathrm{PTS}}=10^{-3}$ at $S N R=24 d B$ are obtained for $I B O=6 d B \quad$ whereas $B E R_{\mathrm{MC}-\mathrm{CDMA} \text { original with } \mathrm{HPA}}=$ $2.10^{-3}$ at $S N R=24 d B$ for $I B O=6 d B$.

\section{CONCLUSION}

In the present work, a new method has been presented to reduce the PAPR in MC-CDMA systems. Moreover, calculating complexity of the new system is improved. This technique combines the PTS and SLM techniques and does not need IFFT subblocks. Simulation results were achieved by comparing the studied method to the original system in term of BER and CCDF. Obtained results showed that the proposed method is less complex and exhibits significant performance in term BER and CCDF.

\section{REFERENCES}

[1] Y. H. Kim, I. Song, S. Yoon, and S. R. Park, "A multicarrier CDMA system with adaptive subchannel allocation for forward links," IEEE Transactions on Vehicular Technology, vol. 48, no. 5, pp. 1428-1436, 1999.

[2] S. Ragusa, J. Palicot, Y. Louët, and C. Lereau, "Invertible clipping for increasing the power efficiency of OFDM amplification," 2006.

[3] H. I. D. Wasaff, "Adaptive pre-distortion for nonlinear high power amplifiers in OFDM systems," Universitat Politècnica de Catalunya, 2004.

[4] B. Sarala, D. S. Venkateswarulu, and B. N. Bhandari, "Overview of mc cdma PAPR reduction techniques," Arxiv Preprint Arxiv, 1204.3874, 2012

[5] I. Baig, M. Ayaz, and V. Jeoti, "A SLM based localized SC-FDMA uplink system with reduced PAPR for LTE-A," Journal of King Saud University-Engineering Sciences, vol. 25, no. 2, pp. 119-123, 2013.

[6] Y. Xiao, M. Chen, F. Li, J. Tang, Y. Liu, and L. Chen, "PAPR reduction based on chaos combined with SLM technique in optical OFDM IM/DD system," Optical Fiber Technology, vol. 21, pp. 81-86, 2015.

[7] J. Xia, Y. Li, Z. Zhang, M. Wang, W. Yu, and S. Wang, "A suboptimal TR algorithm with fixed phase rotation for PAPR reduction in
MC-CDMA system," in Proc. IET International Conference on Information and Communications Technologies (IETICT 2013), pp. 415-420, 2013

[8] H. Merah, D. Slimani, and M. F. Alsharekh, "PAPR reduction in SFBC-MIMO-MC-CDMA systems using method of attenuation complex chips," in Proc. IEEE 3rd International Conference on Control, Engineering and Information Technology (CEIT), pp. 1-5, 2015.

[9] M. García-Otero and L. A. Paredes-Hernández, "PAPR reduction in SFBC MIMO MC-CDMA systems via user reservation," EURASIP Journal on Advances in Signal Processing, vol. 2011, no. 1, pp. 1-10, 2011.

[10] R. VanNee and R. Prasad, OFDM for Wireless Multimedia Communications, Boston, USA: Artech House, 2000.

[11] C. Rapp, "Effects of the HPA-nonlinearity on a 4-DPSK/OFDM signal for a digital sound broadcasting system," in Proc. ECSC'91, vol. 1, pp. 179-184, Oct. 1991

[12] L. Yang, K. K. Soo, S. Q. Li, and Y. M. Siu, "PAPR reduction using low complexity PTS to construct of OFDM signals without side information," IEEE Transactions on Broadcasting, vol. 57, no. 2, pp. 284-290, 2011.

[13] N. Taspnar, D. Karaboğa, M. Yıldırım, and B. Akay, "Partial transmit sequences based on artificial bee colony algorithm for peak-to-average power ratio reduction in multicarrier code division multiple access systes," Communications, IET, vol. 5, no. 8, pp. 1155-1162, 2011.

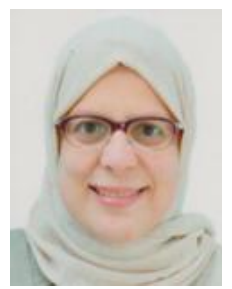

Dr. Mesri Mokhtaria was born in Oran on 20th June 1968. In 2009 she received master degree in in educational sciences (very good plus title of promotion major), professional specialty, UTICEF, University of Strasbourg, France. In 2013 she received very honorable doctorate in electrical engineering, Algeria. In 2014 she received English skills certificates. In 2015 she received HDR in electrical engineering.

She is vice rector in charge of External Relations and Cooperation, UAT Laghouat, Algeria, to current date. She is vice dean of external relations and scientific research, UAT Laghouat, Algeria, until January 2013. She is also responsible for the option 'Signal Processing, UAT Laghouat, Algeria,until July 2011. Coordinator and member of current Erasmus Plus Programs. She has been Certified Reviewer in many International Indexed Journals.

She is mainly skilled in: ESP (English for specific purposes), signal theory/ signal processing, matlab programming, modeling solar energy, mathematics and others. 